\title{
Optical binding in nanoparticle assembly: Potential energy landscapes
}

\author{
Justo Rodríguez, Luciana C. Dávila Romero, and David L. Andrews \\ School of Chemical Sciences, University of East Anglia, Norwich, NR4 7TJ, United Kingdom
}

(Received 23 June 2008; published 3 October 2008)

\begin{abstract}
Optical binding is an optomechanical effect exhibited by systems of micro- and nanoparticles, suitably irradiated with off-resonance laser light. Physically distinct from standing-wave and other forms of holographic optical traps, the phenomenon arises as a result of an interparticle coupling with individual radiation modes, leading to optically induced modifications to Casmir-Polder interactions. To better understand how this mechanism leads to the observed assemblies and formation of patterns in nanoparticles, we develop a theory in terms of optically induced energy landscapes exhibiting the three-dimensional form of the potential energy field. It is shown in detail that the positioning and magnitude of local energy maxima and minima depend on the configuration of each particle pair, with regards to the polarization and wave vector of the laser light. The analysis reveals how the positioning of local minima determines the energetically most favorable locations for the addition of a third particle to each equilibrium pair. It is also demonstrated how the result of such an addition subtly modifies the energy landscape that will, in turn, determine the optimum location for further particle additions. As such, this development represents a rigorous and general formulation of the theory, paving the way toward full comprehension of nanoparticle assembly based on optical binding.
\end{abstract}

DOI: 10.1103/PhysRevA.78.043805 PACS number(s): 42.50.Wk, 37.10.Vz, 78.67.Bf, 81.16.Rf

\section{INTRODUCTION}

It was first observed by Ashkin [1] that the transverse spatial gradient in the irradiance of a laser beam may be used to accelerate and trap particles. This finding has inspired investigations and applications of optical manipulation to particles ranging from cold atoms to living cells [2-5], and it has led to the rapid development of optical tweezers, a tool that has since been extensively developed for the manipulation of mesoscopic particles [6]. In laser configurations allowing more than one particle to be trapped, it also emerged that optically modified interparticle interactions may be observed. The influence of laser light on particle interactions was demonstrated theoretically, using quantum electrodynamics [7], and the effects were first observed experimentally through studies on pairs of micrometer-sized particles, using optical tweezers [8]. The recognition of these forces and determination of means for their control has opened up a considerable diversity of methods for the fabrication of micro- and nanoparticle arrays.

Optically induced interparticle interactions (usually known as optical binding, although the forces are not necessarily attractive in form) have been a particular focus in extensive recent investigations [9-27]. The phenomenon has increasingly been advocated as a tool for optical manipulation of particles, and many optically induced arrays and configurations have been observed experimentally [25,27-29]. Laboratory studies are commonly performed with counterpropagating beams, where possible complications due to light pressure are obviated and where particles, trapped by gradient forces, are organized by optical binding forces in the overlapped region of maximum beam irradiance [13]. In other investigations, the traps are much larger in dimension than the typical particle separations, and the observed particle organization is entirely attributable to the optical binding potentials [16].
Classically, optical binding may be understood as an interaction of individual particles with the electromagnetic fields generated by light scattering and optical rectification processes in other particles. A directly corresponding quantum electrodynamical representation casts the mechanism in terms of four photon events: the annihilation of an input photon within one particle, mediation of the interaction between this and another particle through the propagation of virtual photons (created at one particle, annihilated at the other), and the stimulated reemission of an input-mode photon in either of these particles. By either method, the interparticle potential can be generated and evaluated using perturbation theory [30]. Recent research has shown that the potential energy oscillates with particle separation, generating complex patterns of maxima and minima that are capable of supporting a variety of two-dimensional arrays [21-23].

In the following, we briefly review the methodology for these calculations, and we reveal new multiparticle features exhibited by the potential. In Sec. II, an expression for the optically induced pair potential, for an interaction between particles of any shape, is derived using quantum electrodynamics (QED). Developed using the electric-dipole approximation, the potential significance of multipolar contributions is also assessed. In Sec. III, by locating local energy minima, we determine the geometric configurations that may arise for two spherical particles in a plane wave. Section IV focuses on the rolling form of the optically induced landscapes that emerge. The analysis reveals how the positioning of local minima determines the energetically most favorable locations for the addition of a third particle to each such pair. In Sec. V, the analysis is then extended to three-particle systems. It is shown how successive modifications to the energy landscape, resulting from each particle addition, determine the optimum locations for subsequent particle additions. In Sec. VI, we conclude with a consideration of optically conferred interactions between molecular aggregates and the dielectric effect of a host medium. 


\section{OPTICALLY INDUCED PAIR POTENTIAL ENERGY: QED}

In previous work [9], based on QED, it has been shown that the origins of optical binding lie in a form of radiationinduced coupling representing an extension to the usual Casimir-Polder interactions, the latter signifying dispersion interactions in a form which accommodate the effects of retardation. In the absence of populated radiative modes, Casimir-Polder interactions entail the pairwise exchange of virtual photons [31] - i.e., two virtual photon creation events and two corresponding annihilations. (The reader is referred elsewhere [32] for a concise alternative QED representation, also based on QED, but closer in spirit to the classical representation.) When laser light is present, one or more radiation modes have a nonzero occupation number and any photon from these modes may take the place of one virtual photon, generating intensity-dependent additional terms. In this section a concise summary of the ensuing calculation is given.

The interactions of the electromagnetic field with functionally identical particles $(A, B)$ are fundamentally based on electric interactions with the radiation field, expressible as follows (using the implied summation convention for repeated tensor and vector indices):

$$
\begin{aligned}
H_{\mathrm{int}}^{\xi}= & -\varepsilon_{0}^{-1} \sum_{\xi=A, B} \mu_{i}(\xi) d_{i}^{\perp}\left(\mathbf{R}_{\xi}\right) \\
& -\varepsilon_{0}^{-1} \sum_{\xi=A, B} Q_{i j}(\xi) \nabla_{j} d_{i}^{\perp}\left(\mathbf{R}_{\xi}\right)-\cdots,
\end{aligned}
$$

$\mathbf{R}_{\xi}$ being the position vector of a dielectric particle $\xi$, with $\boldsymbol{\mu}(\xi)$ and $\mathbf{Q}(\xi)$ the corresponding operators for the electric dipole and electric quadrupole ( $E 1$ and $E 2$, respectively). The potential involvement of coupling with the magnetic field of the radiation is to be briefly discussed later in this section. The second term in (1), which for radiation in the uv or visible range is typically smaller than the first by the order of the fine structure constant (i.e., two to three orders of magnitude smaller), takes the lead in a series of higher-order multipole corrections; for the present, only this leading correction is retained, with a view to the subsequent analysis. The electric displacement vector field $\mathbf{d}^{\perp}$ in (1) is itself expressible, using the Power-Zienau-Woolley approach, as a mode expansion that is linear in the photon creation and annihilation operators [30].

For any two particles $A$ and $B$, a suitable basis set for calculating the optical coupling, induced by throughput radiation, comprises products of the electronic states of each particle and a number state for each mode of the radiation field; the particle pair energies emerge as diagonal elements of the perturbation matrix. It follows from above that the leading contributor to optically induced coupling can be described by four entangled $E 1$ interactions: the annihilation and creation events of one real and of one virtual photon. No lower order that is diagonal in the basis set can serve to causally couple the two particles and to engage throughput radiation. Since each operation of the electromagnetic field entails a photon creation or annihilation, the leading term in the system energy is delivered by fourth-order perturbation theory. After performing lengthy calculations and casting the result in terms of physically measurable quantities, the following result emerges for the dominant term in the optically induced shift in interparticle potential energy, produced by a beam of irradiance $I[33]$ :

$$
\begin{aligned}
\Delta E_{\text {ind }}(\mathbf{k}, \mathbf{R})= & \left(\frac{I}{\varepsilon_{0} c}\right) e_{i}^{(\lambda)} \bar{e}_{l}^{(\lambda)} \operatorname{Re}\left\{\alpha_{i j}^{A}(k) V_{j k}(k, \mathbf{R}) \alpha_{k l}^{B}(k)\right. \\
& \times \exp (-i \mathbf{k} \cdot \mathbf{R})+\alpha_{i j}^{B}(k) V_{j k}(k, \mathbf{R}) \alpha_{k l}^{A}(k) \\
& \times \exp (i \mathbf{k} \cdot \mathbf{R})+\frac{1}{2} V_{j k}(0, \mathbf{R})\left[\beta_{i j l}^{A}(k) \mu_{k}^{B}\right. \\
& \left.\left.+\mu_{k}^{A} \beta_{i j l}^{B}(k)\right]\right\},
\end{aligned}
$$

where $\mathbf{R}$ is the separation between the particles, $\mathbf{R}_{B}-\mathbf{R}_{A}, e_{i}^{(\lambda)}$ is the $i$ th component of the laser polarization vector, $\alpha_{i j}^{\xi}(k)$ $\equiv \alpha_{i j}^{\xi}(-k ; k)$ is the frequency-dependent polarizability and $\beta_{i j l}^{\xi}(k) \equiv \beta_{i j l}^{\xi}(-k ; 0, k)$ the corresponding electro-optic hyperpolarizability of particle $\xi[30]$, and $V_{j k}(k, \mathbf{R})$ is the retarded resonance dipole-dipole interaction tensor [35],

$$
\begin{aligned}
V_{i j}(k, \mathbf{R})= & \frac{\exp (i k R)}{4 \pi \varepsilon_{0} R^{3}}\left[(1-i k R)\left(\delta_{i j}-3 \hat{R}_{i} \hat{R}_{j}\right)\right. \\
& \left.-(k R)^{2}\left(\delta_{i j}-\hat{R}_{i} \hat{R}_{j}\right)\right] .
\end{aligned}
$$

No other contributions arise at this order $\left(E 1^{4}\right)$. The calculation that leads to (3) involves a summation over radiative modes for a virtual photon traveling in either direction between $A$ and $B$; however, it is assumed that the real photon annihilation and creation processes occur specifically at $A$ and $B$, respectively. The last two terms in Eq. (2) involve static dipole moments $\boldsymbol{\mu}$ for each particle; each term relates to a process in which both the annihilation and recreation of the beam photons are colocated at the other particle. Clearly, if both particles are centrosymmetric and nonpolar as will be assumed in the following, then these terms vanish.

Before proceeding further we now address higher multipolar contributions to the induced energy shift $\Delta E_{\text {ind }}(\mathbf{k}, \mathbf{R})$. The next highest-order contributions emerge when the second term in Eq. (1), the electric-quadrupole interaction Hamiltonian, is considered as the coupling basis for any one of the four photon events described previously. Introducing the appropriate modification to the first two terms in Eq. (2), the additional $\left(E 1^{3} E 2\right)$ contributions to the energy which result are given by

$$
\begin{aligned}
\Delta E_{\text {ind }}^{(Q)}(k, \mathbf{R})= & \left(\frac{I}{\varepsilon_{0} c}\right) \operatorname{Re}\left(e _ { i } ^ { ( \lambda ) } \overline { e } _ { l } ^ { ( \lambda ) } \left\{i V _ { j k } ( k , \mathbf { R } ) k _ { m } \left[A_{i m j}^{A}(k) \alpha_{k l}^{B}(k)\right.\right.\right. \\
& \left.-\alpha_{i j}^{A}(k) A_{k m l}^{\prime B}(k)\right]+\alpha_{i j}^{A}(k) V_{j k m}(k, \mathbf{R}) A_{k m l}^{\prime B}(k) \\
& \left.\left.+A_{i j m}^{A}(k) V_{j m k}(k, \mathbf{R}) \alpha_{k l}^{B}(k)\right\} \exp (-i \mathbf{k} \cdot \mathbf{R})\right)
\end{aligned}
$$

Here, the term involving the dipole-dipole interaction tensor $V_{j k}(k, \mathbf{R})$ arises from contributions where one of the real laser photon interactions is accomplished by an electricquadrupole interaction. In the remainder of the above expression (4), it is the virtual coupling photon that experiences one quadrupole interaction, reflected in the involvement of the 
fully retarded resonance quadrupole-dipole interaction tensor $V_{i j k}(k, \mathbf{R})$, given by [36]

$$
\begin{aligned}
V_{i j k}(k, \mathbf{R})= & \frac{i e^{i k R}}{4 \pi \varepsilon_{0}}\left[\left(\hat{R}_{i} \hat{R}_{j} \hat{R}_{k}-\delta_{i j} \hat{R}_{k}\right)\left(\frac{k^{3}}{R}+\frac{i k^{2}}{R^{2}}\right)+\left(\delta_{i j} \hat{R}_{k}\right.\right. \\
& \left.\left.+\delta_{i k} \hat{R}_{j}+\delta_{j k} \hat{R}_{i}-5 \hat{R}_{i} \hat{R}_{j} \hat{R}_{k}\right)\left(\frac{i k^{2}}{R^{2}}+\frac{3 k}{R^{3}}+\frac{i 3}{R^{4}}\right)\right] .
\end{aligned}
$$

The frequency-dependent dipole-quadrupole polarizabilities $A_{i m k}^{\xi}(k)$ and $A_{i j k}^{\prime \xi}(k)$ for particle $\xi$ are expressible as

$$
\begin{aligned}
& A_{i m k}^{\xi}(k)=\sum_{s}\left[\frac{\mu_{i}^{0 s}(\xi) Q_{m k}^{s 0}(\xi)}{\left(E_{s 0}-\hbar c k\right)}+\frac{Q_{m k}^{0 s}(\xi) \mu_{i}^{s 0}(\xi)}{\left(E_{s 0}+\hbar c k\right)}\right], \\
& A_{i j k}^{\prime \xi}(k)=\sum_{s}\left[\frac{Q_{i j}^{0 s}(\xi) \mu_{k}^{s 0}(\xi)}{\left(E_{s 0}-\hbar c k\right)}+\frac{\mu_{k}^{0 s}(\xi) Q_{i j}^{s 0}(\xi)}{\left(E_{s 0}+\hbar c k\right)}\right] .
\end{aligned}
$$

In passing we note that the energy contributions given by Eq. (4) (whose symmetry conditions are to be discussed below) are not of a form previously identified as the lowest-order correction to the all-dipole result. In extensive studies of optical binding forces by Salam [37], generalized formulas have been presented for the pair energy of particles with electric polarizabilities of arbitrary multipole order. The assumption has been that each excited state, featured in the sum over states in the generalized polarizability equations, connects with the ground state only through one particular order of multipole. Hence the general form of those results is an $E m^{2} E n^{2}$ shift for arbitrary integers $m$ and $n$. The expression obtained in Eq. (3.2) of Ref. [37] and given as the lowest-order correction is therefore of $E 1^{2} E 2^{2}$ form; i.e., it is the result calculated for a case involving two electricquadrupole events. However, symmetry permitting, expression (4) is the lowest-order correction to the energy because it invokes only one quadrupole interaction.

The odd-parity rank-3 tensors $\mathbf{A}^{\xi}$ and $\mathbf{A}^{\prime \xi}$ vanish if the particles are centrosymmetric. Specifically, the only nonzero components of these tensors are those which transform under the totally symmetric representation of the appropriate molecular point or space group [34]. Both tensors, on reduction into irreducible parts, prove to carry weight- $1,-2$, and -3 contributions (by the rule of angular momentum addition for the $E 1$ and $E 2$ transition moments which they each entail). Crucially, any dipolar particle (i.e., one whose static electricdipole moment is nonzero, requiring odd-parity weight-1 components to transform under the totally symmetric representation) will also satisfy the symmetry criteria for oddparity weight-3 components to be allowed. For the same symmetry reason, however, it proves that such particles will also have nonzero $\boldsymbol{\beta}^{\xi}$ tensors. Hence, the third and fourth terms in Eq. (2) will also contribute, and since they have $E 1^{4}$ form, they will certainly outweigh any contribution involving an electric quadrupole. For completeness, it is also important to note that the leading corrections produced by the involvement of a magnetic-dipole interaction are of the same

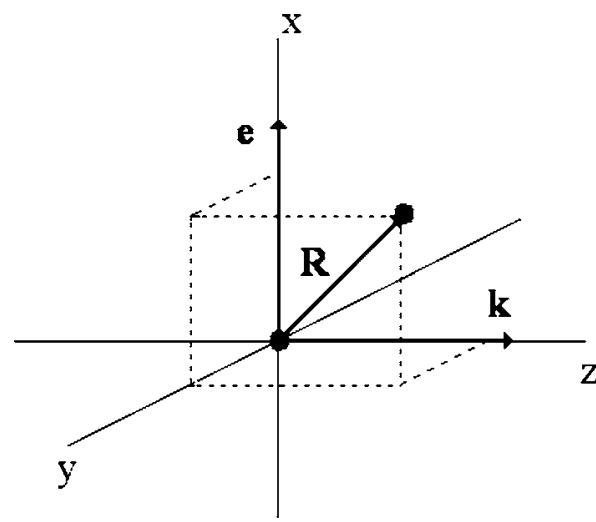

FIG. 1. Two particles in a plane wave of polarization e and wave vector $\mathbf{k}$. The vector $\mathbf{R}$ denotes the displacement between the particles $A$ and $B$, where $A$ can be considered as located at the origin. The $x$ and $z$ axes are defined along $\mathbf{e}$ and $\mathbf{k}$, respectively.

order as the electric-quadrupole correction [34] and therefore they, too, can be disregarded in the following study of the optically induced interaction energy shift.

In the following sections we begin to focus discussion on the optically induced potential directly expressed in terms of molecular properties. It is to be borne in mind that, when nanoparticles are considered, the magnitude of the potential depends on bulk quantities; these are to be taken into account, together with the dielectric effect of the medium, in Sec. VI.

\section{GEOMETRIC CONFIGURATION OF PARTICLES AND FIELDS}

Previous studies of the optically induced interparticle potential energy landscapes for spherical particles in a single plane wave have revealed intricate features conducive to the production of a variety of attractive and repulsive forces, and torques, engendering the formation of several multiparticle structures [21-23]. Here, we use a different parametrization that is shown to reveal additional features in the potential energy. The Cartesian coordinates are defined as shown in Fig. 1; the Poynting vector is along the $z$ axis and linear polarization along the $x$ axis. Applying Eq. (2) to $N$ spherical particles, the total energy shift may be written as

$$
\begin{aligned}
\Delta E= & \frac{1}{2} \sum_{\xi=1}^{N} \sum_{\xi^{\prime}=1}^{N}\left(\frac{2 I \alpha^{2} k^{3}}{4 \pi \varepsilon_{0}^{2} c\left(k R^{\left(\xi \xi^{\prime}\right)}\right)^{3}}\right)\left\{\left(\cos k R^{\left(\xi \xi^{\prime}\right)}\right.\right. \\
& \left.+k R^{\left(\xi \xi^{\prime}\right)} \sin k R^{\left(\xi \xi^{\prime}\right)}\right)\left[1-3\left(\hat{R}_{x}^{\left(\xi \xi^{\prime}\right)}\right)^{2}\right] \\
& \left.-\left(k R^{\left(\xi \xi^{\prime}\right)}\right)^{2} \cos k R\left[1-\left(\hat{R}_{x}^{\left(\xi \xi^{\prime}\right)}\right)^{2}\right]\right\} \cos \left(k R_{z}^{\left(\xi \xi^{\prime}\right)}\right),
\end{aligned}
$$

where $\mathbf{R}^{\left(\xi \xi^{\prime}\right)}=\left(R_{x}^{\left(\xi \xi^{\prime}\right)}, R_{y}^{\left(\xi \xi^{\prime}\right)}, R_{z}^{\left(\xi \xi^{\prime}\right)}\right)$ is the displacement vector from particle $\xi$ to particle $\xi^{\prime}$, and $\hat{R}_{x}^{\left(\xi \xi^{\prime}\right)}$ is the projection on the $x$ axis of a unit vector on the direction of $\mathbf{R}^{\left(\xi \xi^{\prime}\right)}$.

When considering cylindrically symmetric particles, variables corresponding to additional degrees of freedom need to be introduced to describe the orientation of the particle axes; 
a suitable parametrization for the optically induced interaction force between two nanotubes has been the subject of previous studies [38]. Of course, even more degrees of freedom need to be included to describe completely anisotropic particles. However, under equilibrium conditions when the only external field is the one associated with the throughput laser radiation, the largest polarizability component of every particle is expected to be aligned with the polarization of the beam, if the particles are well separated, so that they do not exert a significant influence on each other's individual orientation. Under these conditions, only the diagonal (principal axis) elements of the polarizability tensor, on the framework illustrated in Fig. 1, need be considered in the summation of Eq. (2). Moreover, due to the orientation of the polarization on the $x$ axis, only one component contributes. Consequently, the result for cylindrically symmetric particles is again delivered by Eq. (8), where $\alpha$ is now to be interpreted as the largest polarizability element, usually the one along the cylindrical axis.

\section{OPTICALLY INDUCED LANDSCAPES}

In most optical binding applications it is possible to regard the laser irradiance as being essentially time invariant, the characteristic time scale for intensity fluctuations being small compared to those involved in the particle dynamics. Even if pulsed radiation is used in an experiment, the pulse repetition interval is in general sufficiently short, compared to the particle diffusion times, that the input operates as being effectively constant in time. In such an environment, particles are individually displaced by gradient forces to the neighboring maxima of irradiance in the beam structure, known as optical traps, the latter being identifiable with the beam axis in the simplest Gaussian profile case. The location of any such optical trap signifies a relatively static environment, one that facilitates the resolution and measurement of any optically induced pair forces. A further simplifying factor is that these traps are generally much larger in dimension than the typical particle separations, and therefore the applied irradiance may also be taken to be approximately spatially constant, making the optically induced interparticle potential entirely separable from the gradient forces. It can also be assumed that the separation of the particles is sufficiently large-and the laser pulses intense enough - to justify neglect of the Casimir-Polder potential with respect to its laserinduced counterpart; this assumption is also expected to apply in most cases, when particle separations are of the order of the laser wavelength. Under this combination of conditions, applicable in the majority of experiments, the optically induced interparticle potential operates to translate particles towards separations that correspond to its own energy minima.

An analysis of the spatial dependence of the optical binding forces reveals the arrangement of particles in an optical trap. Assuming spherical particles, landscapes of the potential energy surfaces generated by the optically induced pair forces can be exhibited as a function of the Cartesian components of the separation vectors, $R_{x}, R_{y}$, and $R_{z}$, as shown in Fig. 2. The distance scales on this and subsequent graphs are
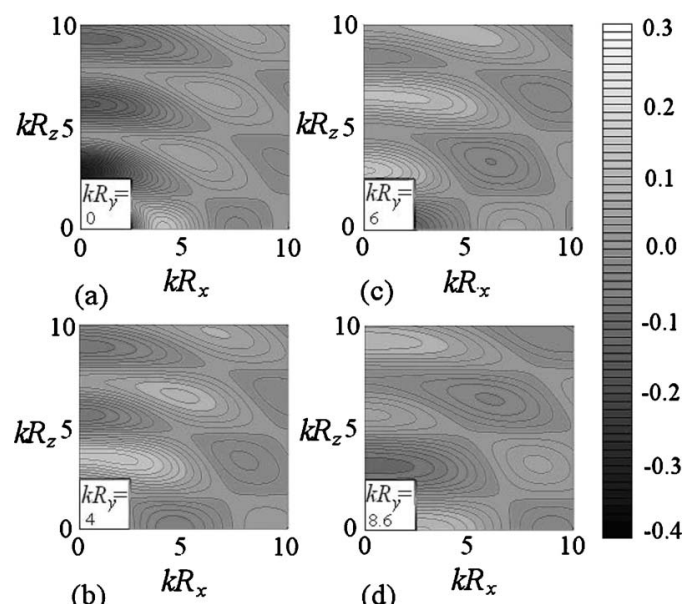

FIG. 2. Optically induced interparticle energy surfaces as a function of the interparticle displacement vector $\mathbf{R}=\left(R_{x}, R_{y}, R_{z}\right)$ for different values of $k R_{x}$. The scale is in $2 I \alpha^{2} k^{3} /\left(4 \pi \varepsilon_{0}^{2} c\right)$ units. Field polarization directed along the $x$ axis, wave vector on the $z$ axis.

normalized by plotting results against dimensionless variables scaled by $k$ (whose value is $2 \pi$ times the inverse wavelength of the applied radiation). One of the interesting features that is immediately evident in Fig. 2(a) is the sequence of minima for particles on the $z$ axis, responsible for longitudinal optical binding. With increasing $R_{y}$, Figs. 2(b)-2(d), the maxima and minima generally exhibit an increase in absolute energy, accompanied by a drift in a counterclockwise direction towards the $(y, z)$ plane (signified by the vertical axis). Thus, particles trapped in any of the energy minima will tend to fall into the $(y, z)$ plane.

It is apparent that these principles afford a tractable basis for extension to a system of $N$ spherical particles, irradiated with a linearly polarized beam. In general, a determination of the optically induced potential experienced by the whole assembly requires the evaluation of Eq. (2) for $N ! /(N-2) ! / 2$ particle pairs, obviously making an analysis of the system significantly more complex when considering a large number of particles. It is interesting and expedient to approach the problem by considering the possibility of optically fabricating structures for the simplest case of three particles, a system that represents the key prototype for developing generic models of multiparticle complexity.

\section{THREE OR MORE PARTICLES}

The energetics associated with the arrangements of more than two particles can now be explored by placing particles in the minima of a suitable template that optimizes the pair interactions. As shown by the analysis in the last section, the landscape shown in Fig. 3 is one that is physically favored, and it provides a sensible basis for determining the optically induced potential energy resulting from placement of one or more additional particles on the $(y, z)$ plane. We begin with the contour maps of the energy surfaces of three interacting particles, as a function of the Cartesian coordinates of one of the particles, as shown in Fig. 4. A number of significant potential energy minima may be observed for any of the 


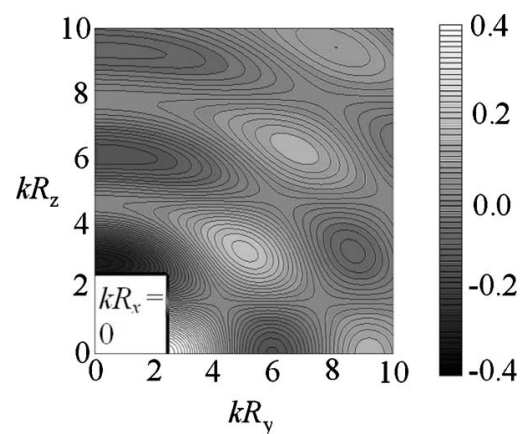

FIG. 3. Optically induced inter particle energy surface on the $(y, z)$ plane of Fig. 1. The scale is in $2 I \alpha^{2} k^{3} /\left(4 \pi \varepsilon_{0}^{2} c\right)$ units. Polarization and wave vector as in Fig. 2.

three surfaces considered, revealing a dramatic increase in the number of stable, physically realistic arrays with increasing number of particles. When the fixed displacement vector is longitudinal to the direction of propagation of the particles and its magnitude is $k R=3$ as in Fig. 4(a), the most significant minima are given by the sequence $(0,3 n, 0)$ for $n$ $=2,3,4, \ldots$ with an energy that is inversely proportional to $n$; the formation of a longitudinal chain is the most stable configuration, as may also be observed in Fig. 4(b) when the magnitude of this separation is $k R=6$. When the displacement between the two fixed particles is transverse to the propagation direction, as in Fig. 4(c), for example, there is a sequence of potential energy minima at approximately the positions $(0,3 n, 3)$ for $n=1,2,3, \ldots$ with the lowest of them for $n=2$; another significant minimum may be observed close to the position $(0,8,3)$. When the fixed displacement vector is given by $(0,8.6,3)$, as in Fig. 4(d), there are two important symmetrically equivalent stability points at approximately $(0,2,3)$ and $(0,7,0)$.

When four particles are present, as in Fig. 5, a significant reduction of the energy shift may be observed over the entire surface; in this case, the most stable position for the fourth

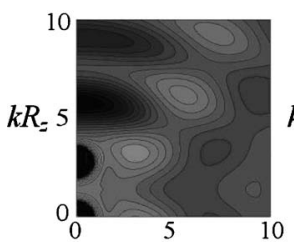

(a) $k R_{v}$

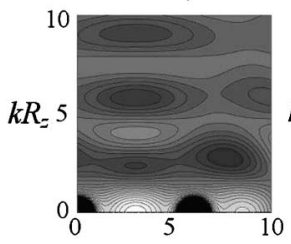

(c)

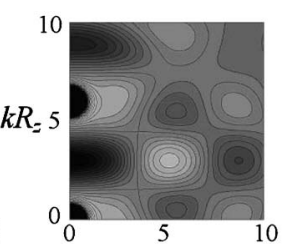

(b) $k R_{v}$

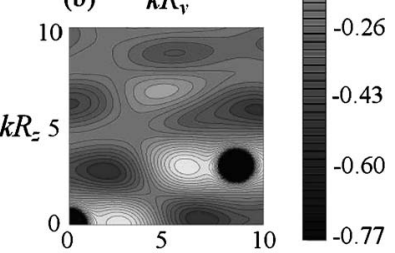

(d) $k R_{1}$

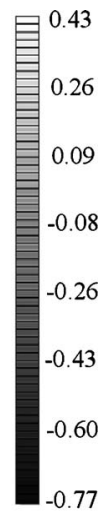

FIG. 4. Optically induced potential energy landscapes for three identical interacting particles as a function of the vector positions of one of them, when the other two are located at the origin and at (a) $(0,0,3)$; (b) $\quad(0,0,6) ; \quad$ (c) $\quad(0,6,0) ; \quad$ (d) $\quad(0,8.6,3)$. Scale in $2 I \alpha^{2} k^{3} /\left(4 \pi \varepsilon_{0}^{2} c\right)$ units. Black circular shapes represent local divergences in energy shift in the proximity of the fixed particles. Polarization and wave vector as in Fig. 2.

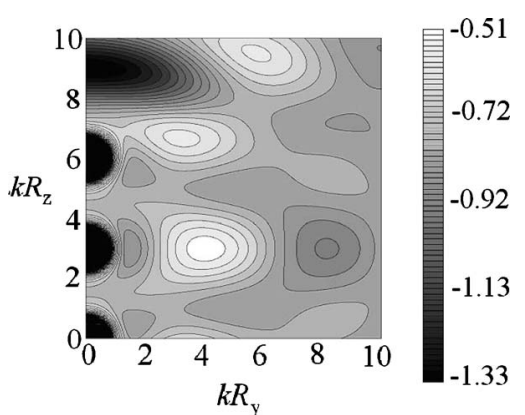

FIG. 5. Optically induced potential energy landscapes for four identical interacting particles as a function of the vector positions of one of them, when the other three are at $(0,0,0),(0,0,3)$, and $(0$, $0,6)$. Scale in $2 I \alpha^{2} k^{3} /\left(4 \pi \varepsilon_{0}^{2} c\right)$ units. Polarization and wave vector as in Fig. 2, black circular shapes as in Fig. 4.

particle is found for the longitudinal arrangement; another important stable configuration is close to $(0,8,3)$. Although the introduction of an additional particle in the system may induce a readjustment of the fixed particles, and some of these structures may be slightly different to those suggested by the contour maps (requiring optimization of the potential energy as a function of the interparticle displacement vectors), it is evident that variety of two-dimensional arrays may be optically fabricated in a plane perpendicular to the polarization of the laser beam.

\section{MOLECULAR AGGREGATES}

To consider optical binding between molecular aggregates, each of which is formed by $p$ molecules or optical centers, we need to entertain an effective polarizability in Eq. (2), given by the expression [34]

$$
\chi_{i j}^{(\zeta)}=\sum_{\xi}^{p} \alpha_{\lambda \mu}^{(\xi)} I_{i \lambda}^{(\xi)} I_{j \mu}^{(\xi)} \exp \left(i \mathbf{k} \cdot \Delta \mathbf{R}_{\xi}\right)
$$

where the $I_{\lambda i}^{(\xi)}$ are the cosine of the angles between the spacefixed axis and the molecule-fixed axis. The relative position of molecule $\xi$ within the aggregate $\zeta$ is given by $\Delta \mathbf{R}_{\xi}$. These aggregates can be considered as mesoscopically disordered material, within which local domains possess particular structures. The optically induced potential takes the form

$$
\begin{aligned}
\Delta E_{\text {ind }}^{\text {total }}\left(k, \mathbf{R}_{B A}\right)= & \left(\frac{I \alpha^{2}}{\varepsilon_{0} c}\right) \operatorname{Re}\left[V_{x x}\left(k, \mathbf{R}_{B A}\right)\right. \\
& \times\left(\sum_{\xi, \xi^{\prime}}^{p, p^{\prime}} \exp \left(i \mathbf{k} \cdot \Delta \mathbf{R}_{\xi, \xi^{\prime}}\right) \exp \left(-i \mathbf{k} \cdot \mathbf{R}_{B A}\right)\right. \\
& \left.\left.+\sum_{\xi, \xi^{\prime}}^{p, p^{\prime}} \exp \left(-i \mathbf{k} \cdot \Delta \mathbf{R}_{\xi, \xi^{\prime}}\right) \exp \left(i \mathbf{k} \cdot \mathbf{R}_{B A}\right)\right)\right],
\end{aligned}
$$

where 


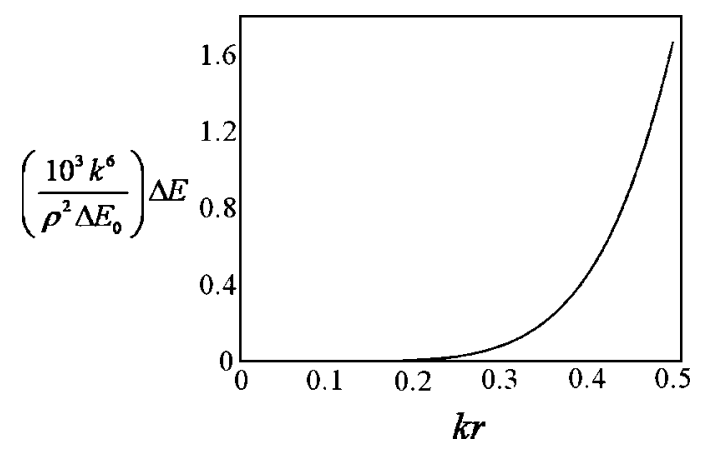

FIG. 6. Optically induced force plotted against nanoparticle radius: $\Delta E_{0}$ is the optically induced force between two molecules, of the same type of those comprising the nanoparticles.

$$
\begin{aligned}
\sum_{\xi, \xi^{\prime}}^{p, p^{\prime}} \exp \left( \pm i \mathbf{k} \cdot \mathbf{R}_{\xi, \xi^{\prime}}\right) & \sim \sum_{\xi}^{p} 1+\sum_{\xi \neq \xi^{\prime}}^{p} \exp \left( \pm i \mathbf{k} \cdot \mathbf{R}_{\xi, \xi^{\prime}}\right) \\
& \sim p+\text { nonlinear term }
\end{aligned}
$$

and $\Delta \mathbf{R}_{\xi, \xi^{\prime}} \equiv \Delta \mathbf{R}_{\xi^{\prime}}-\Delta \mathbf{R}_{\xi^{\prime}}$ The first term signifies a linear contribution, present for particles of any size; it is significant that it persists for large particles where $\Delta \mathbf{R}_{\xi, \xi^{\prime}}$ is several orders of magnitude larger than the optical wavelength $\lambda$ $(=2 \pi / k)$, such as the case of microparticles (see, for example, Ref. [14]). The second term of (11) contributes for small particles where we have $\Delta \mathbf{R}_{\xi, \xi^{\prime}} \ll \lambda$; in this case, the weighting factor is proportional to $p(p-1)$, as in the case of nanoparticles. In the limiting case, using a similar analysis to that given in Ref. [5], the sum over molecules $\xi$ in the susceptibility $\chi_{i j}^{(\xi)}$ may be approximated by a continuous integral. With these assumptions, we can express the optically induced potential between two spherical aggregates of radius $\boldsymbol{r}$ as follows:

$$
\begin{aligned}
\Delta E & =\left(\frac{I \alpha^{2} \rho^{2}}{\varepsilon_{0} c k^{6}}\right)(\sin k r-k r \cos k r)^{2} \operatorname{Re}\left[V_{x x}(k, \mathbf{R})\right] \cos \left(k R_{y}\right) \\
& \equiv \Delta E_{0} \frac{\rho^{2}}{k^{6}}(\sin k r-k r \cos k r)^{2},
\end{aligned}
$$

where $\rho$ is the number density of molecules and $\alpha$ the isotropic molecular polarizability. Representing the dependence on $\rho$ in this manner demonstrates that the effect of optical binding, in aggregates comprising relatively few highvolume particles, will be less acute than in other aggregates of the same size, comprising a larger number of low-volume species. In Fig. 6, where the optically induced potential energy is plotted against particle radius, a dramatic increase of pair energy with the aggregate size parameter $r$ can be observed.

To complete the representation of a standard trapping environment, account can be taken of the dielectric influence of any medium in which the particles are individually suspended. It has previously been shown [15] that the relative values of the refractive index between the particles and the surrounding medium significantly influence optical binding phenomena, modifying the positions of stability. With incorporation of the appropriate Lorenz field factors, the dependence on $k R$ changes to a dependence on $n(c k) k R$, where the multiplier is the complex refractive index properties of the medium supporting the particles. Most experiments are conducted using wavelengths at which the system under study is optically transparent, well away from any resonance. Then, the dielectric effect of the medium is equivalent to a simple scaling of the particle separations by the index $n$; i.e., the results reported and exhibited here can be regarded as establishing the coordinates of potential energy minima on a scale where all distance values are truly given by $R / n$.

\section{CONCLUSION}

In this paper, the theory of optical binding has been developed in a form designed to help elucidate the assembly and formation of patterns in micro- and nanoparticles. It has been revealed how the location and magnitude of potential energy maxima and minima depend on the local configuration of each particle pair, with regards to the polarization and wave vector of the laser light. The study has demonstrated how templates, derived by analysis of the pair energy landscapes, can be used to determine the energetically most favorable locations for the addition of a third and subsequent particles to each equilibrium pair.

To refine the analysis and to take it on to the next stage of development, the next aim is the challenge of identifying and calculating contributions associated with multiparticle processes, involving the entangled near-field interactions of more than two particles. Multiple processes of stimulated scattering have also to be entertained, in order to fully understand the raft of mechanisms involved in the formation of stable particle arrays. The full incorporation of such features in a comprehensive analysis represents our objective and will be subject of future work.

\section{ACKNOWLEDGMENTS}

We thank David Bradshaw and Jamie Leeder for helpful comments. This work is funded by the Engineering and Physical Sciences Research Council.
[1] A. Ashkin, Phys. Rev. Lett. 24, 156 (1970).

[2] M. Greiner, O. Mandel, T. Esslinger, T. W. Hänsch, and I. Bloch, Nature (London) 415, 39 (2002).

[3] D. Meschede and A. Rauschenbeutel, Adv. At., Mol., Opt. Phys. 53, 75 (2006).
[4] K. Dholakia, W. M. Lee, L. Paterson, M. P. MacDonald, R. McDonald, I. Andreev, P. Mthunzi, C. T. A. Brown, R. F. Marchington, and A. C. Riches, in Proceedings of the IEEE Conference Quantum Electronics (IEEE, New York, 2007), p. 1646. 
[5] A. A. Deniz, S. Mukhopadhyay, and E. A. Lemke, J. R. Soc., Interface 5, 15 (2008).

[6] K. Dholakia, P. Reece, and M. Gu, Chem. Soc. Rev. 37, 42 (2008).

[7] T. Thirunamachandran, Mol. Phys. 40, 393 (1980).

[8] M. M. Burns, J.-M. Fournier, and J. A. Golovchenko, Phys. Rev. Lett. 63, 1233 (1989).

[9] D. L. Andrews, R. G. Crisp, and D. S. Bradshaw, J. Phys. B 39, S637 (2006).

[10] D. Maystre and P. Vincent, J. Opt. A, Pure Appl. Opt. 8, 1059 (2006).

[11] T. M. Grzegorczyk, B. A. Kemp, and J. A. Kong, Opt. Lett. 31, 3378 (2006).

[12] T. M. Grzegorczyk, B. A. Kemp, and J. A. Kong, Phys. Rev. Lett. 96, 113903 (2006).

[13] V. Karasek, K. Dholakia, and P. Zemanek, Appl. Phys. B: Lasers Opt. 84, 149 (2006).

[14] F. Chen, G. L. Klimchitskaya, V. M. Mostepanenko, and U. Mohideen, Opt. Express 15, 4823 (2007).

[15] J. Rodríguez, L. C. Dávila Romero, and D. L. Andrews, J. Nanophotonics 1, 019503 (2007).

[16] M. Guillon, O. Moine, and B. Stout, Phys. Rev. Lett. 96, 143902 (2006).

[17] N. K. Metzger, R. F. Marchington, M. Mazilu, R. L. Smith, K. Dholakia, and E. M. Wright, Phys. Rev. Lett. 98, 068102 (2007).

[18] R. G. Crisp and D. L. Andrews, Proc. SPIE 6483, 648304 (2007).

[19] S. Ahlawat, R. Dasgupta, and P. K. Gupta, Proc. SPIE 6535, 65350W (2007).

[20] V. Karasek and P. Zemanek, J. Opt. A, Pure Appl. Opt. 9, S215 (2007).

[21] J. Rodríguez, L. C. Dávila Romero, and D. L. Andrews, Proc.
SPIE 6905, 69050H (2008).

[22] L. C. Dávila Romero, J. Rodríguez, and D. L. Andrews, Opt. Commun. 281, 865 (2008).

[23] L. C. Dávila Romero, J. Rodríguez, and D. L. Andrews, Proc. SPIE 6988, 69880L (2008).

[24] L. C. Dávila Romero and D. L. Andrews, Structured Light and Its Applications: An Introduction to Phase-Structured Beams and Nanoscale Optical Forces, edited by D. L. Andrews (Academic, Burlington, MA, 2008), pp. 79-105.

[25] M. Guillon and B. Stout, Phys. Rev. A 77, 023806 (2008).

[26] M. Li and J. Arlt, Opt. Commun. 281, 135 (2008).

[27] R. F. Marchington, M. Mazilu, S. Kuriakose, V. GarcésChávez, P. J. Reece, T. F. Krauss, M. Gu, and K. Dholakia, Opt. Express 16, 3712 (2008).

[28] S. K. Mohanty, J. T. Andrews, and P. K. Gupta, Opt. Express 12, 2746 (2004).

[29] C. D. Mellor, T. A. Fennerty, and C. D. Bain, Opt. Express 14, 10079 (2006).

[30] D. P. Craig and T. Thirunamachandran, Molecular Quantum Electrodynamics (Dover, Mineola, New York, 1998).

[31] E. A. Power, Introductory Quantum Electrodynamics (Longmans, London, 1964), pp. 34 and 131.

[32] E. A. Power, Eur. J. Phys. 22, 453 (2001).

[33] D. S. Bradshaw and D. L. Andrews, Phys. Rev. A 72, 033816 (2005).

[34] D. L. Andrews and P. Allcock, Optical Harmonics in Molecular Systems (Wiley-VCH, New York, 2002).

[35] G. J. Daniels, R. D. Jenkins, D. S. Bradshaw, and D. L Andrews, J. Chem. Phys. 119, 2264 (2003).

[36] G. D. Scholes and D. L. Andrews, J. Chem. Phys. 107, 5374 (1997).

[37] A. Salam, Phys. Rev. A 73, 013406 (2006).

[38] D. L. Andrews and D. S. Bradshaw, Opt. Lett. 30, 783 (2005). 